\title{
Intra-droplet acoustic particle focusing: simulations and experimental observations
}

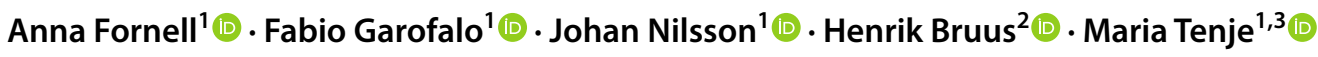

Received: 25 January 2018 / Accepted: 20 June 2018 / Published online: 28 June 2018

(c) The Author(s) 2018

\begin{abstract}
The aim of this paper is to study resonance conditions for acoustic particle focusing inside droplets in two-phase microfluidic systems. A bulk acoustic wave microfluidic chip was designed and fabricated for focusing microparticles inside aqueous droplets (plugs) surrounded by a continuous oil phase in a 380- $\mu \mathrm{m}$-wide channel. The quality of the acoustic particle focusing was investigated by considering the influence of the acoustic properties of the continuous phase in relation to the dispersed phase. To simulate the system and study the acoustic radiation force on the particles inside droplets, a simplified 3D model was used. The resonance conditions and focusing quality were studied for two different cases: (1) the dispersed and continuous phases were acoustically mismatched (water droplets in fluorinated oil) and (2) the dispersed and continuous phases were acoustically matched (water droplets in olive oil). Experimentally, we observed poor acoustic particle focusing inside droplets surrounded by fluorinated oil while good focusing was observed in droplets surrounded by olive oil. The experimental results are supported qualitatively by our simulations. These show that the acoustic properties (density and compressibility) of the dispersed and continuous phases must be matched to generate a strong and homogeneous acoustic field inside the droplet that is suitable for high-quality intra-droplet acoustic particle focusing.
\end{abstract}

Keywords Acoustophoresis · Droplets $\cdot$ Particle manipulation $\cdot$ Two-phase microfluidics $\cdot$ Ultrasound

\section{Introduction}

Manipulation of particles in microfluidic channels using acoustic forces, acoustophoresis, has shown to be a useful tool in a number of lab-on-a-chip applications including the separation of rare cells (Nordin and Laurell 2012; Li et al. 2015), concentration of bacteria (Carugo et al. 2014) and cell trapping (Hammarström et al. 2010; Christakou et al. 2015; Collins et al. 2015). Recently, acoustic particle manipulation has also been implemented in droplet-based microfluidic systems for manipulation of whole droplets (Schmid et al. 2014; Leibacher et al. 2015; Sesen et al. 2015) and

Anna Fornell

anna.fornell@bme.lth.se

1 Department Biomedical Engineering, Lund University, Lund, Sweden

2 Department of Physics, Technical University of Denmark, Kongens Lyngby, Denmark

3 Department Engineering Sciences, Science for Life Laboratory, Uppsala University, Uppsala, Sweden focusing of particles and cells inside droplets (Fornell et al. 2015, 2017; Park et al. 2017).

The interest in droplet microfluidics has emerged because droplets are perfectly suited as miniaturized reaction chambers for encapsulation and investigation of cells at the singlecell level (Schneider et al. 2013; Shembekar et al. 2016), and the technology allows for improved genome engineering and screening of cells (Agresti et al. 2010; Sjostrom et al. 2014). In most of these applications, having the possibility to precisely handle the particles inside the droplets expands the complexity of the assays that can be performed on-chip. Particle manipulation allows, for example, intra-droplet particle enrichment and buffer exchange which are often required in multistep assays (Tenje et al. 2017).

To control the position of particles inside droplets both active and passive methods have been implemented including magnetophoresis (Lombardi and Dittrich 2011; Lee et al. 2014; Brouzes et al. 2015), dielectrophoresis (Han et al. 2017), acoustophoresis (Fornell et al. 2015, 2017; Park et al. 2017) and hydrodynamic methods (Kurup and Basu 2012; Sun et al. 2012; Hein et al. 2015). Of these, acoustophoresis has the advantages of being label free, gentle, and operated in 
non-contact mode. However, in our previous work, there have been experimental indications that the acoustic particle focusing inside droplets is impaired by a difference in acoustic properties between the droplet and the continuous phase (Fornell et al. 2015). Typically, water-based solutions are used as the dispersed phase as living cells are often encapsulated inside the droplets, and fluorinated oils such as Novec HFE-7500 or FC-40 with the addition of fluorosurfactants are used as the continuous phase (also known as the carrier oil). These oils are preferred in droplet-based applications as they allow for the generation of stable water droplets and low cross-contamination between individual droplets, as well as having high gas solubility which is essential for cell viability and proliferation (Baret 2012). However, these oils have very different acoustic properties compared with water, and that is thought to have a negative impact on intra-droplet acoustic particle focusing in two-phase microfluidic systems.

The interaction of sound waves and liquid interfaces has been studied for a long time in both micro- and macrosystems. Almost 80 years ago, the deformation of the interface between two immiscible liquids with different acoustic properties caused by a directed ultrasonic beam was studied (Hertz and Mende 1939). Additionally, the acoustic forces can also act on liquid interfaces in miscible systems, and it has been shown that laminated liquids with different acoustic properties exposed to a standing wavefield can be relocated or stabilized within a microfluidic channel (Deshmukh et al. 2014).

Motivated by these observations and studies, this paper investigates acoustic particle focusing in two-phase systems and in particular how the acoustic properties of the continuous phase influence the acoustic radiation force on particles encapsulated inside droplets. The study is restricted to two cases: water droplets (plugs) generated in fluorinated oil and vegetable olive oil, respectively. Fluorinated oil is chosen since this oil type is commonly used in droplet microfluidic systems, and olive oil is chosen since it has similar acoustic properties as water. First, a simplified 3D model is set up and the acoustic pressure field and the acoustic radiation force on the encapsulated particles is calculated. Second, acoustic particle focusing is analyzed experimentally. This study provides a wider understanding of the fundamental physical principles of intra-droplet acoustic particle focusing, which is essential for the development of optimal system design for acoustic particle manipulation in two-phase microfluidic systems.

\section{Theory and numerical simulations}

\subsection{Acoustophoresis}

Ultrasonic standing waves can be used to position particles in microfluidic channels. For particles larger than $2 \mu \mathrm{m}$ suspended in water, the acoustic boundary layer and viscosity in the $\mathrm{MHz}$ range can be neglected. In this case, the acoustic velocity field $v_{1}$ is given by the gradient of the acoustic pressure field $p_{1}$ as $\boldsymbol{v}_{1}=\frac{-i}{\omega \rho_{\mathrm{wa}}} \nabla p_{1}$, and the acoustic radiation force $\boldsymbol{F}^{\mathrm{rad}}$ acting on the microparticle is a gradient force given by (Bruus 2012)

$$
\begin{aligned}
\boldsymbol{F}^{\mathrm{rad}} & =-\nabla U^{\mathrm{rad}}, \quad \text { with } U^{\mathrm{rad}}=\pi a_{\mathrm{P}}^{3}\left(\frac{f_{0}}{3} \kappa_{\mathrm{WA}}\left|p_{1}\right|^{2}-\frac{f_{1}}{2} \rho_{\mathrm{WA}}\left|\boldsymbol{v}_{1}\right|^{2}\right), \\
f_{0} & =1-\frac{\kappa_{\mathrm{P}}}{\kappa_{\mathrm{WA}}}, \quad \text { and } f_{1}=\frac{2\left(\rho_{\mathrm{P}}-\rho_{\mathrm{WA}}\right)}{2 \rho_{\mathrm{P}}+\rho_{\mathrm{WA}}} .
\end{aligned}
$$

Here $\omega$ is the angular frequency, $f_{0}$ and $f_{1}$ are the monopole and dipole coefficients, $a_{\mathrm{P}}, \rho_{\mathrm{P}}$, and $\kappa_{\mathrm{P}}$ are the radius, density and compressibility of the microparticle, and $\rho_{\mathrm{WA}}$ and $\kappa_{\mathrm{WA}}$ are the density and compressibility of water, respectively. As seen in Eq. (1), the acoustic properties of the particle relative to the surrounding water determine the direction of the radiation force on the particle: a particle with high density and low compressibility is focused to the pressure node whereas a particle with low density and high compressibility is focused to the pressure anti-node. Both plastic particles and cells in water have such acoustic properties that they are focused to the pressure node in an acoustic standing wavefield. The focusing time $t_{\text {foc }}$, defined as the time it takes a particle to move from $y=-\frac{7}{16} W$ to $y=-\frac{1}{16} W$ or $\sim 40 \%$ of the half channel width $W$, is given in terms of the particle radius $a_{\mathrm{p}}$, viscosity $\eta$, and the acoustic energy density $E_{\mathrm{ac}}$ as (Bruus 2012)

$t_{\text {foc }}=\frac{9}{\left(4 f_{0}+6 f_{1}\right) \pi^{2}} \frac{W^{2}}{a_{\mathrm{p}}^{2}} \frac{\eta}{E_{\mathrm{ac}}} \ln \left[\frac{\tan \left(\frac{7}{16} \pi\right)}{\tan \left(\frac{1}{16} \pi\right)}\right]$.

\subsection{Model system used in the numerical simulations}

The system is modelled by considering a water-in-oil droplet confined in a rectangular channel of height $H=100 \mu \mathrm{m}$ and width $W=380 \mu \mathrm{m}$ in an acoustically hard siliconPyrex chip to which the ultrasound is supplied by oscillating the bottom plane with a frequency near $2 \mathrm{MHz}$, see Fig. 1.

While the width $W$ and height $H$ of the channel as well as the height of the silicon and Pyrex layers equal the experimental values, the width $W_{\mathrm{s}}$ of the solid chip and the length $L_{\mathrm{S}}$ of the system has been reduced to enable the demanding 3D simulation on the available 128-GB RAM computer. A further reduction is achieved by limiting the study to systems with rectangular channels that are symmetric around the vertical $y$-z-plane, such that only half of the system needs to be simulated. Moreover, we neglect the thin film of the continuous phase between the droplet and the channel walls (Baroud 


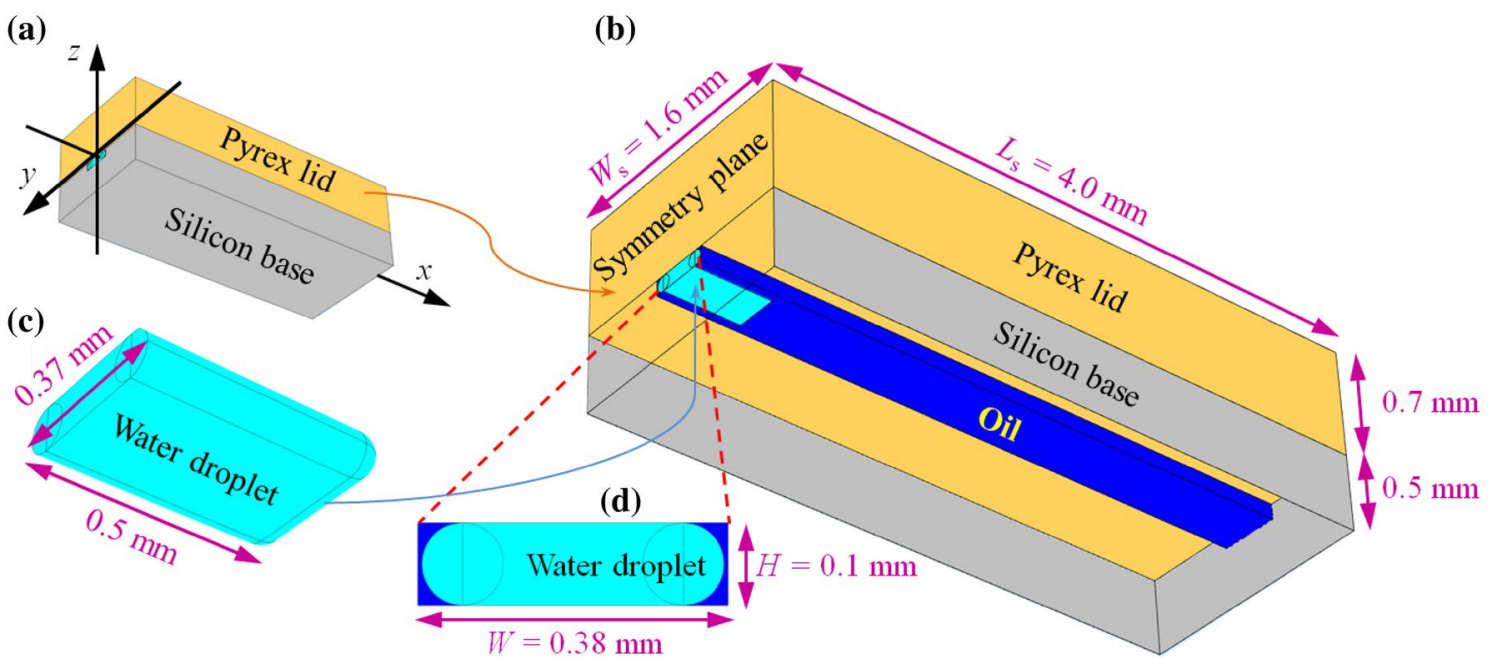

Fig. $13 \mathrm{D}$ model of the microfluidic device used in the numerical simulations. a A sketch of the chip with its 100-oriented single-crystal silicon base (gray) and Pyrex lid (beige). The device is assumed to be symmetric around the $y$-z-plane, such that only half of the system $(x>0)$ needs to be simulated. b The detailed geometry of the device is shown with the silicon layer opened up to make the water

et al. 2010). The material and geometrical parameters used in the simulations are listed in Table 1.

\subsection{Numerical simulations: method}

Simulations of the systems are performed using the finiteelement solver COMSOL Multiphysics (version 5.2) using the method of Ley and Bruus (Ley and Bruus 2017) for Pyrex-water systems, extended to include the single-crystal silicon base using the method of Dual and Schwarz (Dual and Schwarz 2012). The acoustic field is calculated numerically using the linear elastic equations for the displacement field $\boldsymbol{u}_{1}$ in the solid wall with density $\rho_{\mathrm{s}}$, damping coefficient $\Gamma$ and stress tensor $\sigma_{1}$ coupled to the pressure field $p_{1}$ governed by the Helmholtz wave equation in the two fluids (a) and (b),

$-\rho_{\mathrm{s}} \omega^{2}\left(1+i \Gamma_{\mathrm{s}}\right) \boldsymbol{u}_{1}=\nabla \cdot \boldsymbol{\sigma}_{1}$,

$\nabla^{2} p_{1}^{(\mathrm{a})}=-\left(1+i \Gamma_{\mathrm{a}}\right) \frac{\omega^{2}}{c_{\mathrm{a}}^{2}} p_{1}^{(\mathrm{a})}$,

$\nabla^{2} p_{1}^{(\mathrm{b})}=-\left(1+i \Gamma_{\mathrm{b}}\right) \frac{\omega^{2}}{c_{\mathrm{b}}^{2}} p_{1}^{(\mathrm{b})}$

To mimic the non-symmetric actuation of the experiment on the bottom of the silicon base, a harmonically oscillating vertical displacement of the form $\boldsymbol{u}_{1}=d_{1}\left(\frac{1}{2}+\frac{y}{W}\right) e^{-i \omega t} \boldsymbol{e}_{z}$ for droplet (cyan) visible in the long, straight, rectangular oil-filled channel (blue). c The detailed geometry of the flattened water droplet. d End view in the symmetry plane of the water droplet (cyan) and surrounding oil (blue). The bottom plane is actuated vertically as $\boldsymbol{u}_{1}=d_{1}(1 / 2+y / W) e^{-i \omega t} \boldsymbol{e}_{z}$ with a frequency near $2 \mathrm{MHz}$

$-\frac{1}{2} W<y<\frac{1}{2} W$ is imposed, with $\mathrm{d}_{1}=0.4 \mathrm{~nm}$ and using complex-time notation. Here $\omega=2 \pi f$, where $\omega$ and $f$ are the angular frequency and frequency, respectively. On the vertical $y$-z-planes at $x=0$ and $x=L$, we use symmetry boundary conditions (hard wall conditions), and all other outer boundaries have the no-stress condition. On the internal solid-liquid and liquid-liquid interfaces, the boundary conditions are continuity of the normal component of the stress and of the displacement velocities. The corresponding tangential stresses are set to zero. In summary,

Solid - fluid interface : $\boldsymbol{\sigma}_{1} \cdot \boldsymbol{n}=-p_{1} \boldsymbol{n}$ (stress) and

$$
\omega \boldsymbol{n} \cdot \boldsymbol{u}_{1}=\frac{1}{\omega \rho_{\mathrm{f}}} \mathbf{n} \cdot \nabla p_{1} \text { (velocity), }
$$

Fluid $^{(\mathrm{a})}-$ fluid $^{(\mathrm{b})}: p_{1}^{(\mathrm{a})}=p_{1}^{(\mathrm{b})}$ (pressure) and

$$
\frac{1}{\omega \rho_{\mathrm{f}}^{(\mathrm{a})}} \mathbf{n} \cdot \nabla p_{1}^{(\mathrm{a})}=\frac{1}{\omega \rho_{\mathrm{f}}^{(\mathrm{b})}} \mathbf{n} \cdot \nabla p_{1}^{(\mathrm{b})}(\text { velocity }),
$$

Solid - air boundary $: \boldsymbol{\sigma}_{1} \cdot \boldsymbol{n}=0$,

Actuation boundary : $\boldsymbol{u}_{1}=d_{1}\left(\frac{1}{2}+\frac{y}{W}\right) e^{-i \omega t} \boldsymbol{e}_{z}$,

where $\boldsymbol{n}$ is the interface normal vector. We performed a standard numerical convergence study (Ley and Bruus 2017) and found the fields to have converged within a relative deviation of $1 \%$. 
Table 1 Material and geometrical parameters used in the modeling of the droplet systems

\begin{tabular}{|c|c|c|c|}
\hline \multicolumn{2}{|l|}{ Parameters for the solids } & \multicolumn{2}{|l|}{ Parameters for the fluids } \\
\hline Chip base height, $H_{\mathrm{b}}$ & $0.5 \mathrm{~mm}$ & Water density ${ }^{\mathrm{c}}, \rho_{\mathrm{WA}}$ & $997 \mathrm{~kg} \mathrm{~m}^{-3}$ \\
\hline Chip lid height, $H_{1}$ & $0.7 \mathrm{~mm}$ & Water viscosity ${ }^{\mathrm{a}}, \eta_{\mathrm{WA}}$ & $0.89 \mathrm{mPa} \mathrm{s}$ \\
\hline Chip width, $W_{\mathrm{s}}$ & $1.6 \mathrm{~mm}$ & Water sound speed ${ }^{\mathrm{c}}, c_{\mathrm{WA}}$ & $1497 \mathrm{~m} \mathrm{~s}^{-1}$ \\
\hline Chip length, $L_{\mathrm{s}}$ & $8.0 \mathrm{~mm}$ & Fluorinated oil density ${ }^{\mathrm{c}}, \rho_{\mathrm{HFE}}$ & $1621 \mathrm{~kg} \mathrm{~m}^{-3}$ \\
\hline Channel height, $H$ & $0.1 \mathrm{~mm}$ & Fluorinated oil viscosity ${ }^{\mathrm{d}}, \eta_{\mathrm{HFE}}$ & $1.24 \mathrm{mPa} \mathrm{s}$ \\
\hline Channel width, $W$ & $0.38 \mathrm{~mm}$ & Fluorinated oil sound speed ${ }^{\mathrm{c}}, c_{\mathrm{HFE}}$ & $659 \mathrm{~m} \mathrm{~s}^{-1}$ \\
\hline Pyrex density ${ }^{\mathrm{a}}, \rho_{\text {py }}$ & $2230 \mathrm{~kg} \mathrm{~m}^{-3}$ & Olive oil density ${ }^{\mathrm{c}}, \rho_{\mathrm{OLV}}$ & $910 \mathrm{~kg} \mathrm{~m}^{-3}$ \\
\hline Pyrex longitudinal sound speed ${ }^{\mathrm{a}}, c_{\mathrm{L}, \mathrm{py}}$ & $5592 \mathrm{~m} \mathrm{~s}^{-1}$ & Olive oil sound speed ${ }^{\mathrm{c}}, c_{\mathrm{OLV}}$ & $1450 \mathrm{~m} \mathrm{~s}^{-1}$ \\
\hline Pyrex transverse sound speed ${ }^{\mathrm{a}}, c_{\mathrm{T}, \mathrm{py}}$ & $3424 \mathrm{~m} \mathrm{~s}^{-1}$ & Olive oil viscosity ${ }^{\mathrm{e}}, \eta_{\mathrm{OLV}}$ & $60.8 \mathrm{mPa} \mathrm{s}$ \\
\hline Silicon density ${ }^{\mathrm{b}}, \rho_{\mathrm{si}}$ & $2329 \mathrm{~kg} \mathrm{~m}^{-3}$ & Damping coefficient for all fluids ${ }^{\mathrm{a}}, \Gamma_{\mathrm{f}}$ & 0.004 \\
\hline Silicon elastic constant ${ }^{\mathrm{b}}, C_{11}$ & $165.7 \mathrm{GPa}$ & Droplet height, $H_{\mathrm{d}}$ & $0.1 \mathrm{~mm}$ \\
\hline Silicon elastic constant ${ }^{\mathrm{b}}, C_{12}$ & $63.9 \mathrm{GPa}$ & Droplet width, $W_{\mathrm{d}}$ & $0.37 \mathrm{~mm}$ \\
\hline Silicon elastic constant ${ }^{\mathrm{b}}, C_{44}$ & $79.6 \mathrm{GPa}$ & Droplet length, $L_{\mathrm{d}}$ & $1.0 \mathrm{~mm}$ \\
\hline \multirow[t]{4}{*}{ Damping coefficient all solids ${ }^{\mathrm{a}}, \Gamma_{\mathrm{s}}$} & 0.001 & Parameters for $10-\mu \mathrm{m}$-diameter polystyrene test particles & \\
\hline & & Acoustophoretic mobility, $\mu_{\mathrm{ac}}$ & $12\left(\mu \mathrm{m} \mathrm{s}^{-1}\right) / \mathrm{pN}$ \\
\hline & & Buoyancy-corrected gravity force, $F_{\text {grav }}$ & $0.26 \mathrm{pN}$ \\
\hline & & Sedimenting time $H=100 \mu \mathrm{m}, t_{\text {sed }}$ & $32 \mathrm{~s}$ \\
\hline
\end{tabular}

${ }^{a}$ Values from Ley and Bruus (2017)

${ }^{b}$ Values from Hopcroft et al. (2010)

${ }^{\mathrm{c}}$ Measured with Density and Sound Velocity Meter (DSA 5000M, Anton Paar)

${ }^{\mathrm{d}}$ Data sheet from the manufacturer (3M)

${ }^{\mathrm{e}}$ Measured with falling ball microviscometer (Minivis II, Grabner Instruments)

Because the size of the chip in the numerical model is smaller than the actual chip, we first characterize the system with air in the channel to locate the resonances determined solely by the geometry of the solid. In the range from 1.5 to $2.1 \mathrm{MHz}$ we find only two resonances, namely at 1.60 and at $2.01 \mathrm{MHz}$, as shown in Fig. 2, where the black line shows the average acoustic energy density $E_{\mathrm{ac}}^{\text {solid }}$ versus frequency $f$. In the subsequent analysis, with liquid in the channel, we then make sure to avoid these resonances by working in the range from 1.64 to $1.96 \mathrm{MHz}$. Our numerical results are thus independent of the specific smaller chip geometry of the model and can be applied to the larger actual chip geometry. The optimal frequency for acoustophoresis inside the droplet is found by locating resonance peaks in plots of the acoustic radiation force $\boldsymbol{F}^{\mathrm{rad}}$ versus frequency $f$ for a $10-\mu \mathrm{m}$-diameter polystyrene test particle averaged over its position in the water droplet, see Eq. (1). Ideally, the vertical component should vanish, $F_{z}^{\mathrm{rad}}=0$, while the horizontal component should be anti-symmetric and pointing towards the $x$-z-plane with as big as magnitude as possible, $\operatorname{sign}(y) F_{y}^{\text {rad }}<0$, where the sign of the $y$-coordinate, $\operatorname{sign}(y)$, is inserted to pick up the anti-symmetry of the radiation force component in the spatial average.

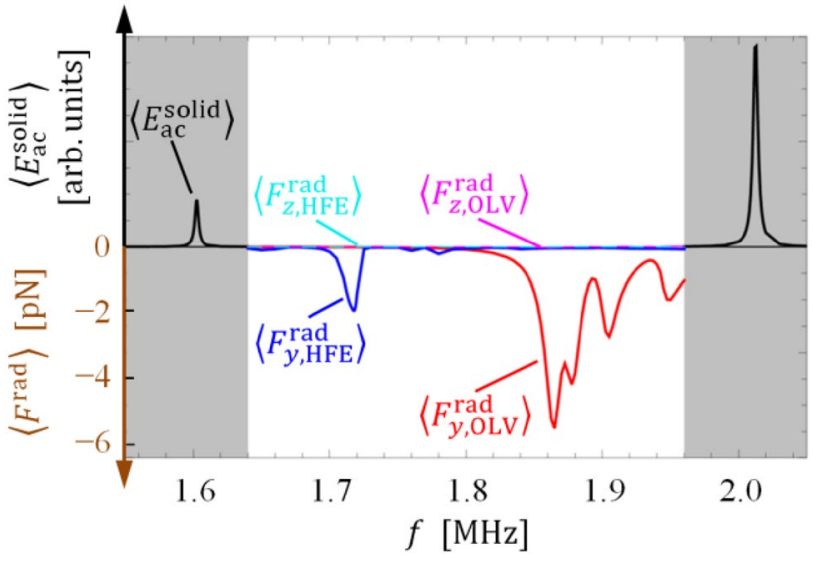

Fig. 2 Resonance curves: averaged acoustic energy density $E_{\mathrm{ac}}^{\text {solid }}$ vs. $f$ in the solid walls for the empty chip (black line), and the averaged $y$ - and $z$-components $F_{y, z}^{\mathrm{rad}}$ of the acoustic radiation force on a $10-\mu \mathrm{m}$-diameter polystyrene test particle in the water droplet in fluorinated oil $\left(F_{y, \mathrm{HFE}}^{\mathrm{rad}}\right.$ blue and $F_{z, \mathrm{HFE}}^{\mathrm{rad}}$ cyan), and olive oil $\left(F_{y, \mathrm{OLV}}^{\mathrm{rad}}\right.$ red and $F_{z, \mathrm{OLV}}^{\mathrm{rad}}$ magneta). The resonances are found to be at $f \stackrel{y, \mathrm{OLV}}{=} 1.718 \mathrm{MHz}$ for fluorinated oil, and at $f=1.865,1.878,1.905$, and $1.950 \mathrm{MHz}$ for olive oil. The latter resonance series corresponds to the number of axial nodes being $0,1,2$, and 3 


\subsection{Numerical simulations: results and discussion}

The resonance curves for a water droplet surrounded by fluorinated oil (marked as HFE) and olive oil (marked as OLV) are shown in Fig. 2. Strong resonances are found at $f=1.718 \mathrm{MHz}$ with $\operatorname{sign}(y) F_{y}^{\mathrm{rad}}=-1.9 \mathrm{pN}$ for a water droplet in fluorinated oil, and at $f=1.865 \mathrm{MHz}$ with $\operatorname{sign}(y) F_{y}^{\mathrm{rad}}=-5.4 \mathrm{pN}$ for a water droplet in olive oil. The vertical components are negligible in the entire frequency range.

A physical interpretation of the resonances and their ability to facilitate acoustophoresis is obtained by studying the acoustic pressure $p$ in the fluids and the displacement $u$ in the solids as well as the acoustic radiation force $\boldsymbol{F}^{\mathrm{rad}}$ in the water droplet for the main resonances at $f=1.718 \mathrm{MHz}$ (fluorinated oil) and at $f=1.865 \mathrm{MHz}$ (olive oil) shown in Fig. 3a, b, respectively.

We first note that for an ideal hard-walled system of width $W=380 \mu \mathrm{m}$, a standing one-half wavelength resonance should occur at $f_{\mathrm{WA}}=c_{\mathrm{WA}} /(2 W)=1.970 \mathrm{MHz}$ in water and at $f_{\mathrm{OLV}}=c_{\mathrm{OLV}} /(2 W)=1.907 \mathrm{MHz}$ in olive oil where $c$ is the sound speed, while a standing two-half wavelength resonance should occur at $f_{\mathrm{HFE}}=c_{\mathrm{HFE}} / W=1.734 \mathrm{MHz}$ in fluorinated oil. We have tested this simple prediction with simulations on the system without a water droplet but filled uniformly with either water, fluorinated oil, or olive oil and found the following resonance frequencies numerically: $f_{\mathrm{WA}}^{\text {num }}=1.918 \mathrm{MHz}, f_{\mathrm{HFE}}^{\text {num }}=1.722 \mathrm{MHz}$, and $f_{\mathrm{OLV}}^{\text {num }}=1.864 \mathrm{MHz}$. For a water droplet in fluorinated oil, Fig. 3a, we first of all note that the numerically obtained resonance frequency $f=1.718 \mathrm{MHz}$ is close to the pure fluorinated oil two-half wavelength resonance of $f_{\mathrm{HFE}}^{\text {num }}=1.722 \mathrm{MHz}$. However, we also observe a clear mismatch between the approximate one-half and two-half standing pressure wavelengths in the water droplet and in the fluorinated oil, respectively. This mismatch leads to a zero pressure and a zero radiation force near the front end of the droplet. Not only is the average magnitude of the radiation force lowered by the acoustic mismatch between the water and the surrounding oil, but the suppression of the radiation force near the front end implies that the radiation force cannot counterbalance the drag forces from the internal circulation flow roll that occurs as the droplet moves inside that channel, forcing the particles away from the center plane (Ohlin et al. 2017). In conclusion, the simulation predicts low-quality acoustophoresis in water droplet surrounded by fluorinated oil. In contrast, the acoustic mismatch between water and olive oil is minute, and we see in Fig. 3b, a nearperfect standing one-half wavelength resonance throughout the fluid domain at $f=1.865 \mathrm{MHz}$, close to the pure olive oil one-half wave resonance $f_{\mathrm{OLV}}^{\text {num }}=1.864 \mathrm{MHz}$, when the water droplet is surrounded by olive oil. As a consequence, there is no suppression of the radiation force near the droplet front end, and the internal flow rolls cannot disperse the particles away from the center axis. In conclusion, the simulation predicts high quality of acoustophoresis in a water droplet surrounded by olive oil.

\section{Experiments}

\subsection{Fabrication of the device}

A silicon-glass microfluidic chip for droplet generation, particle encapsulation, and intra-droplet acoustic particle focusing is fabricated, and in Fig. 4a the chip design is shown schematically.

The microchannels are fabricated using standard photolithography and deep reactive-ion etching on a silicon wafer (500 $\mu \mathrm{m}$ thick, $\langle 100\rangle$-orientation). Deep reactive-ion etching
Fig. 3 a The chip with a water droplet in fluorinated oil at resonance $f=1.718 \mathrm{MHz}$.

The acoustic pressure $p$ from - $156 \mathrm{kPa}$ (blue) to $156 \mathrm{kPa}$ (red) and the magnitude of the acoustic displacement $u$ from $0 \mathrm{~nm}$ (black) to $0.35 \mathrm{~nm}$ (white) in the solids. The inset shows the acoustic radiation force $\boldsymbol{F}^{\mathrm{rad}}$ inside the water droplet with a magnitude from $0 \mathrm{pN}$ (black) to $4.5 \mathrm{pN}$ (white). b Same but for olive oil at resonance $f=1.865 \mathrm{MHz}$ and with amplitudes for $p$ from $-448 \mathrm{kPa}$ (blue) to $447 \mathrm{kPa}$ (red), for $u$ from $0 \mathrm{~nm}$ (black) to $0.35 \mathrm{~nm}$ (white), and for $\boldsymbol{F}^{\mathrm{rad}}$ from $0 \mathrm{pN}$ (black) to $10.7 \mathrm{pN}$ (white) (a)

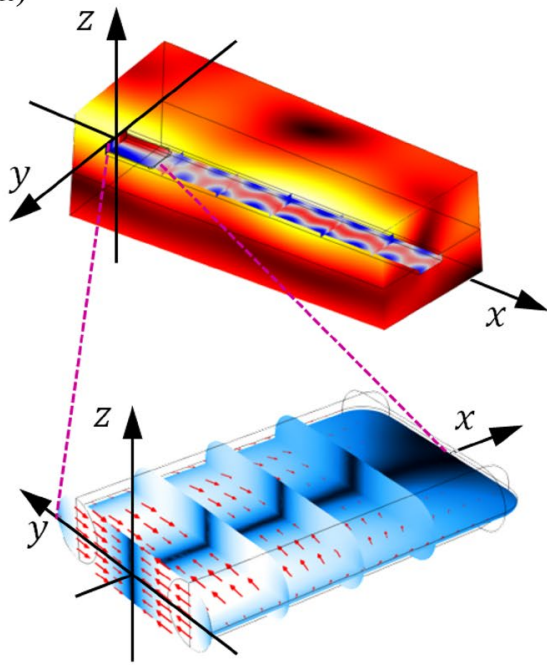

(b)

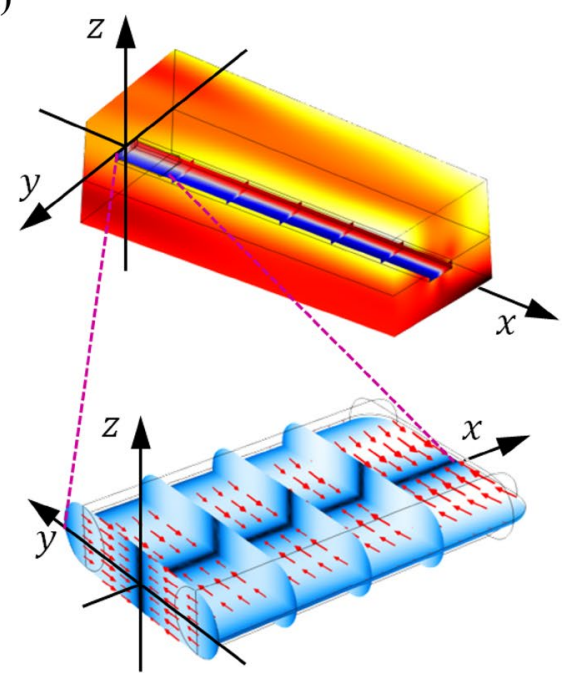


(a)

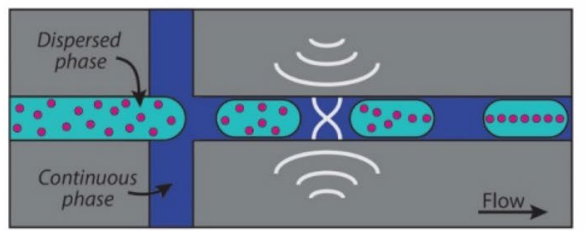

(b)

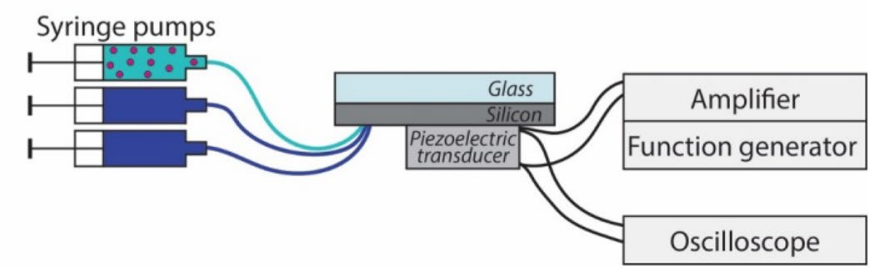

Fig. 4 a Schematic of the microfluidic chip design and $\mathbf{b}$ the experimental setup

is a highly anisotropic etching method that yields channels with rectangular cross sections. The width of the channels is $380 \mu \mathrm{m}$ and the height is $100 \mu \mathrm{m}$. Holes for fluid inlets and outlets are drilled through the silicon wafer. The channels are sealed by anodic bonding of a glass wafer $(700 \mu \mathrm{m}$ thick, Borofloat-33), and the silicon-glass wafer is diced into individual chips. Short pieces of silicone tubings are glued to the chip as fluid connectors. The channels are silanized by flushing the channels with Repel-Silane (Pharmacia Biotech) to make the channels hydrophobic, which is a requirement for generating water-in-oil droplets. To supply the ultrasound, a $12 \mathrm{~mm} \times 12 \mathrm{~mm}$ piezoelectric transducer $(1 \mathrm{~mm}$ thick yielding $2 \mathrm{MHz}$ fundamental resonance frequency, Pz26, Ferroperm Piezoceramics A/S) is glued on the silicon side of the chip using cyanoacrylate glue (Loctite 420, Henkel AG \& Co.), and electrical wires are soldered on the piezoelectric transducer.

\subsection{Experimental method}

The experimental setup is shown in Fig. 4b. The piezoelectric transducer is actuated by a sinusoidal signal from a function generator (33220A, Agilent Technologies Inc.) after amplification of the signal (75A250, Amplifier Research). The output voltage from the function generator is kept constant in the experiments and the voltage over the transducer is monitored using a digital oscilloscope (TDS 1002, Tektronix). The voltage in the experiments over the transducer is between 10 and $25 \mathrm{~V}_{\text {peak-peak }}$ depending on the exact frequency of the ultrasound. The frequency is manually swept between 1.50 and $2.50 \mathrm{MHz}$ in steps of $0.05 \mathrm{MHz}$ to find the strongest particle focusing at the fundamental resonance for each system. The focusing strength is determined by the operator by visually inspecting the system and identifying when the particles are focused using an optical microscope (BX51W1, Olympus) equipped with a $4 \times$ objective. Images are acquired with a camera (XM10, Olympus) mounted on the microscope.

The fluid flows are controlled by three syringe pumps (NEMESYS, Cetoni GmbH) mounted with plastic syringes and connected to the inlet channels via Teflon tubing whereas the outlet channel is kept open. In the experiments, the dispersed phase is Milli-Q water. In the acoustically mismatched system, fluorinated oil (Novec HFE-7500, 3M) containing $2 \%$ surfactant (Krytox FSH-157, Dupont) is used as the continuous phase, and in the acoustically matched droplet system vegetable olive oil (Di Luca and Di Luca) is used as the continuous phase. Microparticles $(10-\mu \mathrm{m}$ polystyrene particles, Sigma-Aldrich) are suspended in the water phase, and in the droplet generation process the particles are encapsulated inside the droplets. The total flow rate is kept constant in the experiments ( $5 \mu \mathrm{L} / \mathrm{min})$ to ensure the residence time of the droplet in the channel is the same. However, the length of the droplets is known to be influenced by the material properties of the fluid phases (Utada et al. 2007; Chen et al. 2014), and in the experiment this effect is partly adjusted for by changing the water:oil flow ratio. For generation of water droplets in olive oil, the flow ratio is set to 2:3 compared with 1:4 for generation of water droplets in fluorinated oil.

\subsection{Experimental results and discussion}

In the experiments, water droplets were generated in fluorinated oil and olive oil, respectively, and polystyrene particles were encapsulated inside the droplets. Without actuation of the ultrasound, the particles were positioned in the entire droplet, see Fig. 5a, c. To focus the encapsulated particles, ultrasound at the fundamental resonance frequency was applied. For each system, the frequency was tuned manually to find the strongest particle focusing frequency. To minimize the influence of variations in the acoustic field arising from external factors such as the gluing of the transducer and differences from the microfabrication process, the same microfluidic chip and the same part of the channel was studied throughout the experimental series.

For water droplets in fluorinated oil no strong focusing was observed when the ultrasound was applied; however, it was noted that at $f=1.70-1.75 \mathrm{MHz}$ the particles were weakly affected by the ultrasound, see Fig. 5b. In contrast, when the water droplets were surrounded by olive oil, it was observed that the encapsulated particles were strongly 
Fig. 5 Photographs of acoustic focusing of microparticles inside water droplets in fluorinated oil and olive oil, respectively. At actuation of the ultrasound at the fundamental resonance, a standing wavefield is created between the channel walls, and the particles are affected by the radiation force. The particles are most strongly focused in the water droplet surrounded by olive oil. The droplets are flowing towards the right in the photographs (a)

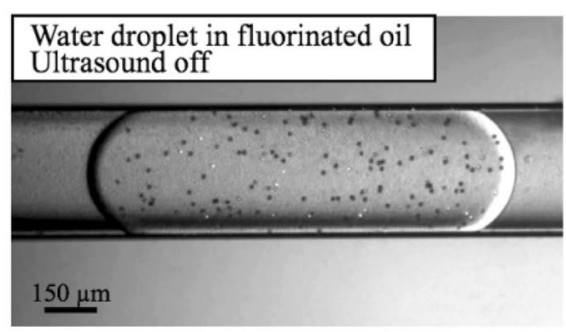

(b)

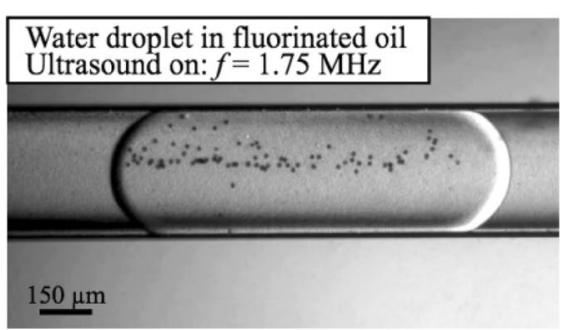

(c)

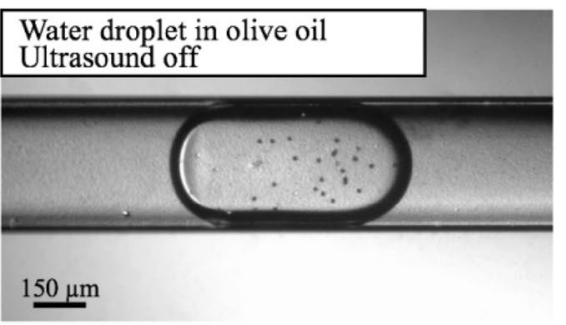

(d)

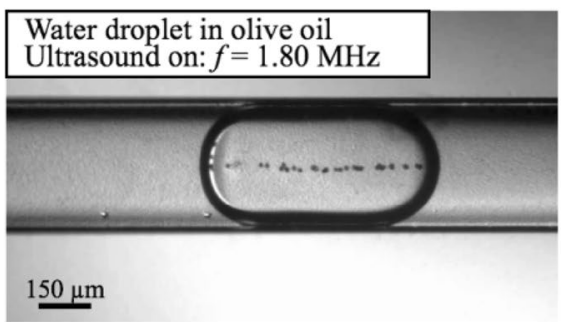

affected by the ultrasound and focused on a very straight line along the center line of the droplets, see Fig. $5 \mathrm{~d}$. The strongest particle focusing was seen at $f=1.70-1.90 \mathrm{MHz}$ for water droplets in olive oil, but some focusing could be observed at higher frequencies, see Table 2 . The fact that the observed frequency range for particle focusing in water droplets surrounded by olive oil was wide indicates that this system is robust and not particularly sensitive to small fabrication and temperature variations, and thus easy and stable to operate.

\section{Concluding discussion}

The numerical simulations and experiments show the importance of matching the acoustic properties of the dispersed and the continuous phase to achieve good quality intra-droplet acoustic particle focusing. Experimentally good particle focusing was only observed for water droplets in olive oil whereas for water droplets in fluorinated oil the significant difference in the acoustic properties between the two phases resulted in weak particle focusing. This can be explained qualitatively from the simulations, which clearly show that strong and uniform focusing occurs in systems with good acoustic matching between the droplet and the continuous phase (olive oil), while focusing is only maintained near the center of droplets having a poor acoustic matching with the continuous phase (fluorinated oil). The lack of focusing forces near the droplet ends is crucial in explaining the poor overall acoustophoretic behavior: as droplets move in microfluidic channels flow rolls are induced inside them (Kinoshita et al. 2007; Ma et al. 2014), and these flow rolls counteract the acoustic focusing force in the front of the droplet (Ohlin et al. 2017). Consequently, in acoustically mismatched droplets, the acoustic focusing is too weak to balance the defocusing Stokes' drag force from the flow rolls, and such droplets are unlikely to exhibit good acoustic particle focusing. Furthermore, in actual experiments, the acoustic fields are never completely perfect and homogeneous, but instead there are areas in the channel with a higher or lower acoustic energy ("hot spots" and "weak spots") due to periodical variations along the length of the channel (Barnkob et al. 2010) as well as bad acoustic coupling, and here the internal flow rolls can have a larger impact and spread the particles more. To quantify the influence from the flow rolls, we note that the fluid flow rolls affect the particles through the Stokes drag force $\boldsymbol{F}^{\mathrm{drag}}=6 \pi \eta a\left(\boldsymbol{v}_{\mathrm{d}}-\boldsymbol{v}_{\mathrm{p}}\right)$, where $\boldsymbol{v}_{\mathrm{d}}$ is the fluid velocity in the droplet and $v_{\mathrm{p}}$ is the particle velocity. In our experiments, the flow rate was $5 \mu \mathrm{L} / \mathrm{min}$, which corresponds to an average fluid velocity of $2 \mathrm{~mm} / \mathrm{s}$. In

Table 2 Measured and simulated resonance frequencies for intra-droplet acoustic particle focusing

\begin{tabular}{lllll}
\hline System & Simulation strong focusing & $\begin{array}{l}\text { Experimental strong } \\
\text { focusing }\end{array}$ & Simulation weak focusing & $\begin{array}{l}\text { Experimental } \\
\text { weak focusing }\end{array}$ \\
\hline Water droplets in fluorinated oil & Not observed & Not observed & $\begin{array}{l}1.72 \mathrm{MHz} \\
\text { above } 1.90 \mathrm{MHz}\end{array}$ & $\begin{array}{l}1.70-1.75 \mathrm{MHz} \\
1.95-2.05 \mathrm{MHz}\end{array}$ \\
Water droplets in olive oil & $1.86-1.90 \mathrm{MHz}$ & $1.70-1.90 \mathrm{MHz}$ & above & \\
\hline
\end{tabular}


rectangular channels, the velocity $\boldsymbol{v}_{\mathrm{d}}$ of the droplets is a factor 0.1-0.4 slower than the fluid velocity, and the relative velocity $v_{\mathrm{d}}-\boldsymbol{v}_{\mathrm{p}}$ is typically another order of magnitude lower (Baroud et al. 2010). We, therefore, estimate the drag force on the particles in our experiment to be of the order $F^{\text {drag }} \approx 2-20 \mathrm{pN}$. Following Barnkob et al. (2010), the magnitude of the radiation force in our experiments is determined by measuring the typical focusing time $t_{\text {foc }}=1 \mathrm{~s}$, which by Eq. (2) gives a typical energy density $E_{\mathrm{ac}}=6 \mathrm{~Pa}$. In the simulations, this value of the energy density is obtained by choosing the actuation amplitude to be $d_{1}=0.4 \mathrm{~nm}$, a value for which the resulting radiation force becomes $F^{\text {rad }} \approx 11 \mathrm{pN}$, or somewhat smaller, as shown in Figs. 2 and 3. Thus, it is within the experimental control of the drag force through the flow rate and of the radiation force through the actuation voltage on the piezocrystal to cross over from a radiation force-dominated to drag force-dominated behavior. However, for a given setting of the control parameters, the focusing ability of the acoustically matched olive oil system is better than for the mismatched fluorinated oil system.

Because the typical timescales for the acoustic focusing of the particles, for the passage of the droplets through the channel, and for the sedimentation of the particles in our experiments are 1,5, and $32 \mathrm{~s}$, respectively, it follows that the influence of gravity is negligible.

The purpose of this study was to investigate how the acoustic properties of the continuous phase affect particle focusing in acoustofluidic two-phase systems; however, from the application viewpoint in addition to suitable acoustic properties, care must also be taken to find fluid phases that provide monodisperse droplet generation, high droplet stability, no cross-contamination and are biocompatible.

\section{Conclusion}

In this work, acoustic focusing of microparticles encapsulated in water-in-oil droplets was studied. Two different systems were considered: one where there was a significant difference in the acoustic properties of the dispersed and continuous phase (water droplets in fluorinated oil) and one where the phases had similar acoustic properties (water droplets in olive oil). The simulations and experimental results show that to obtain high-quality acoustic focusing inside the droplets the acoustic properties of the dispersed and continuous phase should be matched. We believe that these findings will provide important information in designing experiments regarding acoustofluidics in two-phase systems.
Acknowledgements Financial support was provided by the Swedish Research Council, the Crafoord Foundation, Knut and Alice Wallenberg Foundation (Grant no. KAW 2012.0023), Längmanska Kulturfonden, the Royal Physiographic Society of Lund, and Foundation Olle Engkvist Byggmästare.

Open Access This article is distributed under the terms of the Creative Commons Attribution 4.0 International License (http://creativeco mmons.org/licenses/by/4.0/), which permits unrestricted use, distribution, and reproduction in any medium, provided you give appropriate credit to the original author(s) and the source, provide a link to the Creative Commons license, and indicate if changes were made.

\section{References}

3M Data sheet. https://multimedia.3m.com/mws/media/65496 $\mathrm{O} / 3 \mathrm{mtm}$-novectm-7500-engineered-fluid.pdf \& $\mathrm{fn}=$ prodinfo nvc7500.pdf. Accessed 21 May 2018

Agresti JJ, Antipov E, Abate AR et al (2010) Ultrahigh-throughput screening in drop-based microfluidics for directed evolution. Proc Natl Acad Sci 107:4004-4009. https://doi.org/10.1073/ pnas.0910781107

Baret J-C (2012) Surfactants in droplet-based microfluidics. Lab Chip 12:422-433. https://doi.org/10.1039/C1LC20582J

Barnkob R, Augustsson P, Laurell T, Bruus H (2010) Measuring the local pressure amplitude in microchannel acoustophoresis. Lab Chip 10:563-570. https://doi.org/10.1039/b920376a

Baroud CN, Gallaire F, Dangla R (2010) Dynamics of microfluidic droplets. Lab Chip 10:2032-2045

Brouzes E, Kruse T, Kimmerling R, Strey HH (2015) Rapid and continuous magnetic separation in droplet microfluidic devices. Lab Chip 15:908-919. https://doi.org/10.1039/C4LC01327A

Bruus H (2012) Acoustofluidics 7: the acoustic radiation force on small particles. Lab Chip 12:1014-1021. https://doi. org $/ 10.1039 / \mathrm{c} 21 \mathrm{c} 21068 \mathrm{a}$

Carugo D, Octon T, Messaoudi W et al (2014) A thin-reflector microfluidic resonator for continuous-flow concentration of microorganisms: a new approach to water quality analysis using acoustofluidics. Lab Chip 14:3830. https://doi.org/10.1039/ C4LC00577E

Chen X, Glawdel T, Cui N, Ren CL (2014) Model of droplet generation in flow focusing generators operating in the squeezing regime. Microfluid Nanofluidics 1341-1353. https://doi. org/10.1007/s10404-014-1533-5

Christakou AE, Ohlin M, Onfelt B, Wiklund M (2015) Ultrasonic three-dimensional on-chip cell culture for dynamic studies of tumor immune surveillance by natural killer cells. Lab Chip 15:3222-3231. https://doi.org/10.1039/C5LC00436E

Collins DJ, Morahan B, Garcia-Bustos J et al (2015) Two-dimensional single-cell patterning with one cell per well driven by surface acoustic waves. Nat Commun 6:8686. https://doi. org/10.1038/ncomms9686

Deshmukh S, Brzozka Z, Laurell T, Augustsson P (2014) Acoustic radiation forces at liquid interfaces impact the performance of acoustophoresis. Lab Chip 14:3394. https://doi.org/10.1039/ C4LC00572D

Dual J, Schwarz T (2012) Acoustofluidics 3: Continuum mechanics for ultrasonic particle manipulation. Lab Chip 12:244-252. https://doi.org/10.1039/C1LC20837C

Fornell A, Nilsson J, Jonsson L et al (2015) Controlled lateral positioning of microparticles inside droplets using acoustophoresis. Anal Chem 87:10521-10526. https://doi.org/10.1021/acs.analc hem. 5 b02746 
Fornell A, Ohlin M, Garofalo F et al (2017) An intra-droplet particle switch for droplet microfluidics using bulk acoustic waves. Biomicrofluidics 11:31101. https://doi.org/10.1063/1.4984131

Hammarström B, Evander M, Barbeau H et al (2010) Non-contact acoustic cell trapping in disposable glass capillaries. Lab Chip 10:2251-2257. https://doi.org/10.1039/c004504g

Han S, Kim HS, Han A (2017) In-droplet cell concentration using dielectrophoresis. Biosens Bioelectron 97:41-45. https://doi. org/10.1016/j.bios.2017.05.036

Hein M, Moskopp M, Seemann R (2015) Flow field induced particle accumulation inside droplets in rectangular channels. Lab Chip 15:2879-2886. https://doi.org/10.1039/C5LC00420A

Hertz G, Mende H (1939) Der Schallstrahlungsdruck in Flüssigkeiten. Zeitschrift für Phys 114:354-367. https://doi. org/10.1007/BF01337001

Hopcroft MA, Nix WD, Kenny TW (2010) What is the Young' s modulus of silicon? J Microelectromechanical Syst 19:229-238. https ://doi.org/10.1109/JMEMS.2009.2039697

Kinoshita H, Kaneda S, Fujii T, Oshima M (2007) Three-dimensional measurement and visualization of internal flow of a moving droplet using confocal micro-PIV. Lab Chip 7:338-346. https://doi. org $/ 10.1039 / \mathrm{b} 617391 \mathrm{~h}$

Kurup GK, Basu AS (2012) Field-free particle focusing in microfluidic plugs. Biomicrofluidics 6:22008. https://doi.org/10.1063/1.37001 20

Lee H, Xu L, Oh KW (2014) Droplet-based microfluidic washing module for magnetic particle-based assays. Biomicrofluidics 8:44113. https://doi.org/10.1063/1.4892495

Leibacher I, Reichert P, Dual J (2015) Microfluidic droplet handling by bulk acoustic wave (BAW) acoustophoresis. Lab Chip 15:28962905. https://doi.org/10.1039/C5LC00083A

Ley MWH, Bruus H (2017) Three-dimensional numerical modeling of acoustic trapping in glass capillaries. Phys Rev Appl 8:1-15. https://doi.org/10.1103/PhysRevApplied.8.024020

Li P, Mao Z, Peng Z et al (2015) Acoustic separation of circulating tumor cells. Proc Natl Acad Sci USA 112:4970-4975. https://doi. org/10.1073/pnas.1504484112

Lombardi D, Dittrich PS (2011) Droplet microfluidics with magnetic beads: a new tool to investigate drug-protein interactions. Anal Bioanal Chem 399:347-352. https://doi.org/10.1007/s0021 6-010-4302-7

Ma S, Sherwood JM, Huck WTS, Balabani S (2014) On the flow topology inside droplets moving in rectangular microchannels. Lab Chip 14:3611. https://doi.org/10.1039/C4LC00671B
Nordin M, Laurell T (2012) Two-hundredfold volume concentration of dilute cell and particle suspensions using chip integrated multistage acoustophoresis. Lab Chip 12:4610. https://doi.org/10.1039/ c2lc40629b

Ohlin M, Fornell A, Bruus H, Tenje M (2017) Improved positioning and detectability of microparticles in droplet microfluidics using two-dimensional acoustophoresis. J Micromech Microeng 27:84002. https://doi.org/10.1088/1361-6439/aa7967

Park K, Park J, Jung JH et al (2017) In-droplet microparticle separation using travelling surface acoustic wave. Biomicrofluidics 11:64112. https://doi.org/10.1063/1.5010219

Schmid L, Weitz DA, Franke T (2014) Sorting drops and cells with acoustics: acoustic microfluidic fluorescence-activated cell sorter. Lab Chip 14:3710-3718. https://doi.org/10.1039/c4lc00588k

Schneider T, Kreutz J, Chiu DT (2013) The potential impact of droplet microfluidics in biology. Anal Chem 85:3476-3482. https://doi. org/10.1021/ac400257c

Sesen M, Alan T, Neild A (2015) Microfluidic plug steering using surface acoustic waves. Lab Chip 15:3030-3038. https://doi. org/10.1039/C5LC00468C

Shembekar N, Chaipan C, Utharala R, Merten CA (2016) Dropletbased microfluidics in drug discovery, transcriptomics and highthroughput molecular genetics. Lab Chip 16:1314-1331. https:// doi.org/10.1039/C6LC00249H

Sjostrom SL, Bai Y, Huang M et al (2014) High-throughput screening for industrial enzyme production hosts by droplet microfluidics. Lab Chip 14:806-813. https://doi.org/10.1039/C3LC51202A

Sun M, Khan ZS, Vanapalli SA (2012) Blood plasma separation in a long two-phase plug flowing through disposable tubing. Lab Chip 5225-5230. https://doi.org/10.1039/c2lc40544j

Tenje M, Fornell A, Ohlin M, Nilsson J (2017) Particle manipulation methods in droplet microfluidics. Anal Chem. https://doi. org/10.1021/acs.analchem.7b01333

Utada AS, Fernandez-Nieves A, Stone HA, Weitz DA (2007) Dripping to jetting transitions in coflowing liquid streams. Phys Rev Lett 99:1-4. https://doi.org/10.1103/PhysRevLett.99.094502

Publisher's Note Springer Nature remains neutral with regard to jurisdictional claims in published maps and institutional affiliations. 